\title{
Obesidad en personas que viven con trastornos mentales: Revisión del alcance de las estrategias para identificar, prevenir y manejar
}

\author{
Obesity in people living with mental health disorders: Scoping review of strategies to recognize, \\ prevent, and manage
}

Ellis Opusunju ${ }^{1}$, Karen A. Dominguez-Cancino ${ }^{2,3}$, Juan M. Leyva-Moral ${ }^{3,4}$, Nataly J. Membrillo-Pillpe ${ }^{3,5}$, Diane K. Whitehead ${ }^{1}$, Patrick A. Palmieri ${ }^{1,3,6}$

\section{RESUMEN}

Objetivo: Identificar y sintetizar evidencia respecto a las estrategias de prevención y manejo de la obesidad utilizadas para el control de peso de personas que viven con trastornos de salud mental (PVTM) que se encuentran en tratamiento farmacológico. Materiales y Métodos: Diseño: Revisión del alcance. Fuentes de datos: se realizaron búsquedas en las bases de datos CINAHL, Medline, PubMed, Ovid y ProQuest. Método de revisión: se desarrollaron rutas de búsqueda con las palabras clave: obesidad, psiquiatría, salud mental, intervención, manejo, medicamentos, y obesidad. Se identificaron estudios con límite de fecha de publicación de cinco años, publicados en inglés o en español que contaran con resúmenes en inglés. El proyecto de revisión de alcance resultante fue aprobado por el comité de ética. Resultados: se identificaron 60 artículos que abordan la prevención y manejo de obesidad en PVTM. Se reconocieron tres áreas temáticas al considerar la totalidad de los artículos: 1) personas con trastornos mentales graves y obesidad, 2) conocimientos y actitudes de los médicos sobre el tratamiento de la obesidad y, 3) intervenciones preventivas para personas con trastornos mentales. Las PVTM tiene un riesgo elevado de desarrollar obesidad, en conjunto con patologías relacionadas a esta como las enfermedades cardiovasculares. Factores como mala alimentación, baja actividad física, consumo de psicofármacos y vulnerabilidad social pueden ser las causas. El foco de los profesionales de salud mental está centrado en el tratamiento del trastorno mental, dejando de lado temas como la obesidad, abordaje para el cual no se sientes capacitados, a pesar de existir experiencias de intervenciones de reducción de peso exitosas en la literatura. Conclusiones: El entrenamiento de los profesionales debe incluir estrategias para abordar la obesidad en PVTM. Se requiere desarrollar y diseminar guía de práctica clínica basadas en evidencia para el manejo de la obesidad en este grupo de riesgo.

Palabras clave: sobrepeso, obesidad, salud mental, psiquiatría, revisión del alcance, factor social.

\begin{abstract}
Objective: To identify and synthesize evidence regarding obesity prevention and management strategies for the control of the weight of people living with mental health disorders (PLMDs) who are in pharmacological treatment. Materials and Methods: Design: Scoping review. Data sources: Searches in the CINAHL, Medline, PubMed, Ovid and ProQuest databases. Method of revision: Database search with the key words: obesity, psychiatry, mental health, intervention, management, medication and obesity. Studies identified with a five-year publication limit, published in English or Spanish that would have abstracts in English. The project resulting in this scoping review was approved by the university institutional review board. Results: 60 articles were identified that address the prevention and management of obesity in PLMDs. Three thematic areas were recognized when considering all articles: 1) people with mental disorders and obesity, 2) knowledge and attitudes of physicians on the treatment of obesity and 3) preventive interventions for people with mental disorders. PLMDs has a high risk of developing obesity and related pathologies like cardiovascular diseases. Factors such as poor diet, low physical activity, consumption of psychotropic drugs and social vulnerability may be the causes. Mental health professionals are focused on the treatment of mental disorders, leaving aside issues related to obesity, approach for which there are not feeling to have enough trained, although existence of successful experiences on weight reduction interventions in the literature. Conclusions: The training of mental health professionals should include strategies to address obesity in PLMDs. There needs to be increased resources dedicated to developing educational programs for mental health professionals and disseminating evidence-based clinical practice guidelines focused on the management of obesity in this high-risk group.
\end{abstract}

Keywords: Overweight, obesity, mental health, psychiatry, scoping review.

Programa de Doctorado en Enfermería. Facultad de Ciencias de la Salud. Walden University. USA.

${ }^{2}$ Escuela de Enfermería. Universidad Finis Terrae. Chile.

${ }^{3}$ Centro de Investigación EnfAmericas. Asociación Peruana de Enfermería. Lima - Perú.

${ }^{4}$ Departamento de Enfermería. Facultad de Medicina. Universitat Autònoma de Barcelona. España.

${ }^{5}$ Escuela de Enfermería. Universidad María Auxiliadora. Lima - Perú.

${ }^{6}$ Facultad de Ciencias de la Salud. Universidad Privada Norbert Wiener. Lima - Perú. 


\section{INTRODUCCIÓN}

La obesidad es la segunda causa principal de muerte prevenible en los Estados Unidos, considerándose un problema de salud pública. En promedio, una persona obesa tiene 14 años menos de esperanza de vida, en comparación con población general (1). La obesidad se define como una condición donde existe exceso de peso causado por una acumulación excesiva de grasa corporal con efectos negativos en la salud. Esta condición es objetivada a través del Índice de Masa Corporal (IMC), el que corresponde a una forma simple de clasificar a cada persona en las categorías bajo peso $\left(<18 \mathrm{~kg} / \mathrm{m}^{2}\right)$, peso normal $\left(18-24.9 \mathrm{~kg} / \mathrm{m}^{2}\right)$, sobrepeso $\left(25-29.9 \mathrm{~kg} / \mathrm{m}^{2}\right)$ у obesidad $\left(\geq 30 \mathrm{~kg} / \mathrm{m}^{2}\right)$ (1). Una persona se considera obesa cuando existe un exceso de peso del 20\%. En particular, la medida de circunferencia de cintura resulta ser un importante registro ya que el riesgo de presentar enfermedades cardiovasculares $\mathrm{u}$ otras comorbilidades está muy relacionado con la distribución de grasa abdominal (2).

Se estima que uno de cada tres adultos y uno de cada seis niños presentan obesidad en los Estados Unidos (1). La prevalencia de obesidad en la población general en dicho país es de 36,5\%; al analizar la población personas que viven con trastornos mentales (PVTM) esta cifra aumenta a valores entre un $40 \%$ y $60 \%$ (3), con diferencias por sexo, con una prevalencia de $41 \%$ en hombres y cercana a 50\% en mujeres.(4) En Europa Sur las cifras son algo inferiores, probablemente por influencia de la dieta mediterránea (5). En el caso de Italia se ha encontrado una prevalencia de Síndrome Metabólico (incluye obesidad) en PVTM de $26.1 \%$ (6), en Turquía, la cifra se sitúa en $27,7 \%$ para hombres y $39,3 \%$ para mujeres (7) y en el caso de España la prevalencia de síndrome metabólico fue mayor en aquellos pacientes en tratamiento con antipsicóticos $27.0 \%$ (95\% CI, 23.8-30.1\%) vs., población general 14.4\% (14.1$14.6 \%$ ) (8). Los datos anteriormente presentados deben analizarse con cautela. Dentro de las posibles causas que explican las diferencias se encuentra el abuso de los muestreos por conveniencia, la incongruencia entre grupos control e intervención, la inclusión de la obesidad dentro del síndrome metabólico y el uso de datos procedentes únicamente de bases de datos.

Respecto a los factores que favorecen la condición, la evidencia indica una asociación entre factores tales como dietas deficientes y la falta de ejercicio que aumentan la obesidad en PVTM (9). Este dato produce una preocupación importante en términos de salud, ya que la obesidad contribuye al riesgo a presentar patología biliar, diabetes mellitus, osteoartritis, hipertensión, enfermedad coronaria, accidente cerebrovascular y cáncer (3). A medida que avanzan estas condiciones crónicas, aumenta la morbilidad y mortalidad. Se sabe que las PVTM mantienen dietas deficientes, escogen estilos de vida poco saludables, evitan la actividad física y no buscan atención médica de forma regular (4).

Según McKibbin et al. (4), el trastorno bipolar, depresión, esquizofrenia y la ansiedad aumentan el riesgo de obesidad. Estos trastornos mentales también aumentan el riesgo de enfermedades relacionadas con la obesidad, como la diabetes y las enfermedades coronarias. De esta manera el grupo de PVTM se configura como una población de riesgo en relación a estados nutricionales de exceso que expone a altos riesgos de salud, sin embargo, en la práctica clínica psiquiátrica habitualmente no se identifican a las personas con riesgo de obesidad de manera rápida, y tampoco se implementan estrategias de tratamiento basadas en la evidencia (10). El abordaje del problema requiere un enfoque interdisciplinario en la práctica, que incluya enfermeras, dietistas y nutricionistas, para hacer frente a los regímenes de medicamentos que reduzcan al mínimo el aumento de peso, desarrollar programas de ejercicio efectivos y recomendar modificaciones en la dieta. Tales intervenciones oportunas por parte del equipo interdisciplinario pueden prevenir el progreso de la enfermedad y limitar las complicaciones asociadas con la obesidad.

\section{MATERIALES Y MÉTODOS}

La revisión panorámica facilita el desarrollo del conocimiento a través de un proceso estructurado pero flexible para revisar un amplio espectro de evidencia en investigación (11). El proceso inicia con una pregunta de investigación exploratoria (12) dirigida a "determinar conceptos clave, tipos de evidencia y lagunas en la investigación relacionadas con un área o campo definido al buscar, seleccionar y sintetizar sistemáticamente el conocimiento existente" (13). Esta revisión incluyó cinco pasos, recomendados por el marco propuesto por Arksey and O'Malley (14) y revisados por Levac et al. (15). Este proyecto se realizó con la aprobación previa del Comité de Ética de la Walden University (10-1116-0507762). Cada paso en el proceso se analiza con más detalle a continuación.

Paso 1: la pregunta clínica para guiar la revisión se construyó utilizando el marco "Población-Concepto-Contexto (PCC)" 
recomendado por el Joanna Briggs Institute (2015) para las revisiones panorámicas. Esta revisión fue guiada por la pregunta estructurada PCC, “QQué estrategias de prevención y manejo de la obesidad son utilizadas para el control de peso de las personas que viven con trastornos de salud mental en tratamiento farmacológico?"

Paso 2: la estrategia de búsqueda utilizada para esta revisión incluyó tres pasos. En primer lugar, se desarrollaron rutas de búsqueda con las palabras clave: obesidad, psiquiatría, salud mental, intervención, manejo, medicamentos, y obesidad, y operadores booleanos. Segundo, se realizaron búsquedas en las bases de datos CINAHL, Medline, PubMed, Ovid y ProQuest. Tercero, se identificaron estudios con límite de fecha de publicación de cinco años, publicados en inglés o en español que contaran con resúmenes en inglés para ser incluidos en la revisión.

Paso 3: Selección del estudio. El proceso de selección del estudio incluyó tres partes. Primero, se exploraron los títulos para determinar la elegibilidad para la revisión del resumen. Segundo, se revisaron los resúmenes de los títulos incluidos para determinar su elegibilidad y continuar con la revisión completa del artículo. En tercer lugar, los textos completos de los artículos fueron revisados para la inclusión final en esta revisión.

Paso 4: recopilación, organización y evaluación de los datos. Los datos fueron extraídos de cada artículo utilizando una tabla de gráficos desarrollada para registrar información clave. Se aplicó la herramienta de evaluación de la investigación, Práctica basada en la evidencia de enfermería de John Hopkins, a cada trabajo para evaluar el nivel de evidencia, incluyendo una evaluación de los métodos de investigación (16). Los hallazgos se presentaron descriptivamente en la tabla de resumen.

Paso 5: Análisis, síntesis y resumen de los resultados. Aún se encuentra en desarrollo una lista de verificación para informar sobre las revisiones panorámicas por lo que no estuvo disponible para esta revisión. Se utilizó preferentemente elementos de informes para revisiones sistemáticas y extensión de metaanálisis para revisiones panorámicas, o PRISMA$\mathrm{ScR}$ (17).

\section{RESULTADOS}

La búsqueda exploratoria tuvo como resultado 60 artículos publicados en los últimos 5 años; cada artículo destacaba los problemas asociados al manejo de la obesidad en hospitales psiquiátricos y en atención ambulatoria. De los 60 artículos encontrados, 25 estaban enfocados en la prevención de obesidad en personas con patologías mentales y otros 25 en el manejo de obesidad y reducción de peso. Los últimos 10 artículos estaban enfocados en medicamentos utilizados para el tratamiento de patologías psiquiátricas que contribuían al desarrollo de obesidad. Al describir los resultados de esta revisión, los datos se organizan en las siguientes áreas temáticas: 1) Personas con trastornos mentales graves y obesidad, 2) Conocimientos y actitudes de los médicos sobre el tratamiento de la obesidad e 3) Intervenciones preventivas para personas con trastornos mentales. Los resultados se resumen en las siguientes secciones.

\section{Personas con trastornos mentales graves y obesidad}

Galletly y Murray (18) reportan que aproximadamente una de cada 17 personas es diagnosticada con un trastorno mental grave. Durante su vida, las PVTM no sólo presentan una mayor tasa de problemas de salud física, sino que también se enfrentan a discriminación, desempleo y pobreza. Asimismo, experimentan tasas de mortalidad y morbilidad prematura más altas en comparación con la población en general, las PVTM mueren entre 13,5 y 32,5 años antes (19), lo que es explicado en parte debido a su inactividad.(20) Las PVTM tienden a ser menos activas físicamente que los pacientes que no sufren de problemas de salud mental (21).

La obesidad resulta ser más frecuente en las personas con trastornos mentales a diferencia de la población en general (22). Capodaglio, Faintuch y Liuzzi (23) demuestran que el $42 \%$ de los individuos diagnosticados con esquizofrenia presentaban un IMC superior a 27, frente al 27\% de la población general. Yatham y Maj (24) señalan que en personas con trastorno bipolar la tasa de sobrepeso, obesidad y diabetes mellitus es significativamente mayor, en comparación con población general. De forma similar, Yildiz, Ruiz y Nemeroff (25) revisaron 50 estudios de PVTM, concluyendo que la incidencia de obesidad y el sobrepeso eran mayores entre las PVTM en comparación con los pacientes sin trastornos mentales $(59.0 \%$ de obesidad en las PVTM versus el $20.1 \%$ en la población general, y una tasa de sobrepeso del $78.0 \%$ versus $58.2 \%$ en la población general).

Así, la combinación de un trastorno mental, una nutrición deficiente, estilo de vida sedentario, abuso de sustancias, alimentación en exceso y una deficiente coordinación del cuidado contribuyen 
aún más a aumentar el riesgo de un exceso de peso que conduce a la obesidad (26-28). En el estudio de Yildiz et al., el tratamiento con medicamentos, un nivel limitado de ejercicio y el consumo excesivo de carbohidratos fueron las principales causas de obesidad en estos pacientes (25).

La medicación antipsicótica puede ser un factor contribuyente a la elevada prevalencia de la obesidad en PVTM.(28) La prevalencia del síndrome metabólico es mayor en individuos con trastornos mentales; en personas con esquizofrenia, la tasa de prevalencia oscila entre el $40 \%$ y $60 \%$, frente al $27,5 \%$ de la población total, asociándose parte de este aumento al consumo de medicamentos antipsicóticos (21). El consumo de medicamentos no son la única causa de aumento de peso en PVTM, como factores coadyuvantes al consumo de fármacos se ha reconocido al sedentarismo y malos hábitos alimenticios (27).

Udo, Mooney y Newman (29) estudiaron las hospitalizaciones prolongadas en unidades psiquiátricas para establecer el predominio de obesidad y del síndrome metabólico, mostrando que existe una alta prevalencia del síndrome metabólico $(66 \%)$ y obesidad $(33 \%)$ en pacientes psiquiátricos con hospitalización prolongada, hecho que se traduce en impacto serio en la mortalidad y morbilidad física. El mismo estudio sugiere que las PVTM deberían tener acceso a la atención primaria y a otros servicios de salud de manera regular para ayudar a controlar su peso.

Otros factores que contribuyen a la obesidad en las personas con trastornos mentales incluyen el aislamiento social y acceso inadecuado a servicios de salud (27). Cuando estos factores se combinan, contribuyen a elevar el riesgo cardiovascular. Por otra parte, la elección de medicamentos para tratamiento del trastorno mental puede ser un factor de riesgo para las PVTM. A este respecto, Green, Janoff, Yarborough y Yarborough (30) realizaron un programa de intervención de reducción de peso para personas con sobrepeso en tratamiento con antipsicóticos, y establecieron que los efectos adversos podrían contribuir considerablemente a un aumento de la obesidad y trastornos físicos. Además, sostienen que los programas intensivos de control de peso deben ser implementados para prevenir que las personas con trastornos mentales desarrollen obesidad, subrayando que los programas de intervención son necesarios para realzar o mejorar la calidad de vida, ejercen control de los síntomas y reducen las recaídas, así como los reingresos.

\section{Conocimientos y actitudes médicas sobre el tratamiento de la obesidad}

Epling, Morley y Ploutz-Snyder indican que la obesidad ha surgido como una epidemia en la atención primaria, y la mayoría de los médicos de familia parecen no estar seguros de cómo atender a las personas obesas (31). La mayoría de los participantes del estudio reportaron la presencia de barreras para el manejo de la obesidad y la existencia de diferencias entre las creencias sobre las causas de la obesidad, sobre todo entre zonas urbanas y rurales. Los investigadores concluyeron que los enfoques más completos para manejar la obesidad necesitan ser desarrollados por programas médicos educativos.

Existen pocos estudios que permitan entender el problema entre los profesionales de la salud y los pacientes incluidos en programas de reducción de peso. Lichwala-Zyla, Price, Dake, Jordan y Price (32) buscó identificar las percepciones y prácticas médicas $(\mathrm{N}=500)$ relacionadas con el tratamiento y asesoría de los pacientes obesos. Las barreras para el tratamiento fueron frecuentes, incluyendo el cumplimiento deficiente de los pacientes, la falta de guías claras sobre la obesidad, y el temor de ofender a los pacientes. Además, entre los psiquiatras fue frecuente el considerar que su entrenamiento médico era limitado para el manejo de la obesidad, hecho que les dificultaba trabajar con pacientes obesos que buscaban bajar de peso. Sugawara et al. (33) evaluó las actitudes de los psiquiatras japoneses $(n=7.482)$ sobre los eventos adversos relacionados con las anormalidades metabólicas entre los pacientes con esquizofrenia. Los datos indicaron que el $30,5 \%$ de los participantes estaban preocupados por el vínculo entre los medicamentos antipsicóticos y el riesgo de hiperglucemia. Además, el 47,6\% de los participantes reconoció que la frecuencia de monitoreo de la obesidad se basaba en su juicio personal (32).

Según Standford et al., los médicos y otros profesionales de la salud presentan conocimientos limitados para manejar eficazmente la obesidad (34), por su parte, indica que dos tercios de los médicos de atención primaria encuestados se consideraban actores clave en el manejo de la obesidad. Sin embargo, sólo un tercio creía que estaban bien preparados para tratar la obesidad. Además, el $83 \%$ presentó una actitud negativa hacia el tratamiento de pacientes con obesidad. La falta de capacitación, las limitaciones de tiempo y el escaso apoyo administrativo emergieron como los mayores obstáculos en el manejo del sobrepeso y la obesidad (35). 
De acuerdo con Bleich, Bandara, Bennett, Cooper, y Gudzune (36), las perspectivas de los médicos cambian desde su graduación. Los participantes de este estudio (500 médicos de atención primaria) apoyaron la capacitación adicional para los profesionales de la salud, además de cambiar hacia un modelo centrado en la práctica basada en la evidencia para mejorar el manejo clínico de las personas con obesidad. El mismo estudio indica que, aunque la mayoría de los participantes $(97 \%)$ eran conscientes de las causas de la obesidad, desconocían los programas de intervención necesarios para frenar este problema. En consecuencia, las personas con trastornos mentales con obesidad podrían correr un mayor riesgo de muerte prematura en comparación con la población general.

Según Daumit et al. (37), las PVTM reciben poca atención de sus psiquiatras con respecto al aumento de peso y sus problemas asociados. Sin embargo, la implementación de diferentes estrategias de pérdida de peso relacionadas con el comportamiento y su implementación entre los pacientes con trastornos mentales son de gran importancia para todos los profesionales de la salud. Otros autores resaltan la necesidad del trabajo coordinado con los nutricionistas (36). Estas estrategias benefician a las PVTM ya que su peso puede ser controlado y los factores de riesgo para enfermedades crónicas reducidos. El enfoque principal de los psiquiatras en el trabajo con las PVTM es manejar el trastorno mental para mantenerlos involucrados en actividades de la vida diaria (37). Sin embargo, este enfoque selectivo se convierte en un problema a la hora de valorar la salud general de los pacientes, especialmente la del control de peso y obesidad.

Las percepciones y actitudes de los psiquiatras hacia la evaluación y el manejo de la obesidad y su tratamiento no se conocen en profundidad porque no se dispone de estudios publicados sobre el tema (32). Además, las actitudes de los psiquiatras con respecto a los problemas metabólicos siguen siendo en gran parte inexploradas. De esta forma, programas educativos para los psiquiatras que tratan a personas con trastornos mentales serán una forma efectiva de mejorar su práctica y los resultados en los pacientes (33).

La educación de los profesionales de la salud en la gestión de riesgo de la obesidad es muy necesaria para todas las poblaciones (31). Normalmente, los profesionales de la salud suelen estar capacitados, pero cuando evalúan y tratan a los pacientes, sienten que carecen del tiempo necesario para enfatizar los riesgos de la obesidad y explicar las opciones de estilo de vida. El resultado es que los profesionales que trabajan en el área de salud mental en hospitales se centran más en un objetivo psiquiátrico tradicional, como reducir la depresión o ansiedad, en lugar de reducir la obesidad y mejorar las dietas de las PVTM (38). Epling et al. (31) explican que los profesionales de la salud, independientemente del marco de la práctica, necesitan directrices más precisas y mejores instrumentos de detección para identificar y manejar eficazmente la obesidad. Adicionalmente, las barreras para asesorar respecto a la pérdida de peso en la práctica de atención primaria se deben a que los médicos se sienten inadecuadamente preparados en esta área (39). Mazur et al. (40) reportaron que la mayoría de los profesionales de la salud en atención primaria identificaron la necesidad de una educación continua en el manejo de la obesidad.

\section{Estrategias de Prevención y Manejo de la Obesidad}

Brown, Goetz, Hamera y Gajewski (41) señalan que las PVTM requieren programas de intervención para perder peso basados en la evidencia dada la elevada prevalencia de obesidad. La implementación de los programas de intervención debe ser el primer paso para mejorar el bienestar y salud de las PVTM. Es necesario desarrollar programas de intervención en el estilo de vida que fomenten el ejercicio y buenos hábitos alimenticios, los que son eficaces cuando se usan en el tratamiento de la obesidad y el aumento de peso (42). El estudio de Naslund et al. (43) encontró que el 46,9\% de las PVTM estaban tomando medicamentos antipsicóticos de manera regular y, al aumentar de peso, no participaban en un programa de intervención. Después de 12 meses de intervención, se evidenció que, de los participantes del estudio, el 33\% de PVTM y obesidad lograron una pérdida de peso $\geq 5 \%$. Otros participantes del estudio con obesidad severa, pero sin trastornos mentales, lograron una pérdida de peso $\geq 10 \%(20,0 \%)$. Asi, Naslund et al. (43) concluyeron que las PVTM y obesidad severa podrían beneficiarse de participar en intervenciones de modificación y mantenimiento estilos de vida. Brown, Kim, Mitchell e Inskip (44) realizaron una exhaustiva revisión de la literatura para evaluar la eficacia de las intervenciones en los estilos de vida a largo plazo para prevenir el aumento de peso y la obesidad de las PVTM. Estos autores indican que una dieta sana, acompañada de terapia conductual y ejercicio, podría resultar en pérdida significativa de peso y mejora del síndrome metabólico. 
Asimismo, otros estudios sugieren que el tratamiento para la obesidad condujo a una reducción de la diabetes mellitus en el grupo de intervención y en los estilos de vida, en comparación con el grupo control (44). Así, las prácticas de intervención en los estilos de vida son eficaces para reducir el aumento de peso y la tasa de obesidad en PVTM. Además, un enfoque preventivo, que incluye ejercicio físico, resulta un método eficaz y beneficioso para el control del peso y la obesidad (21).

Del mismo modo, Holt et al., realizó un estudio en pacientes coreanos donde se implementó un programa de intervención con una duración de 12 semanas logrando una reducción del IMC de los sujetos. Por su parte, Megna, Schwartz, Siddiqui y Rojas (45) concluyen que la terapia cognitivoconductual producen diferencias significativas en el éxito de la prevención de la obesidad y control del peso. Este resultado se observa especialmente cuando las intervenciones psicológicas se combinan con la actividad física y la dieta. Welsh et al. (46) indican que las diferencias en los procesos cognitivos, motivación, apoyo social y recursos financieros son algunos de los factores que deben ser considerados al adoptar programas de control de peso para los pacientes con problemas de salud mental.

Los profesionales de la salud mental están idealmente posicionados para contribuir en el tratamiento y prevención de la obesidad, pero, como se ha mostrado, carecen de confianza en su capacidad para manejar aspectos de control de peso (47). Sin embargo, los equipos de atención primaria deben convertirse en socios de la implementación de nuevos programas y estrategias de manejo de la obesidad (48). El Grupo de Trabajo de Servicios Preventivos de los Estados Unidos (49), USPSTF, recomendó la implementación de guías clínicas sobre la obesidad basadas en evidencia para todos los proveedores de atención primaria. Estas guías requieren detectar la obesidad en todos los pacientes adultos y proporcionar recomendaciones para el asesoramiento e intervenciones en el comportamiento de las personas con un IMC de 30 o más. El enfoque principal es permitir que los pacientes pierdan peso y manejen su obesidad (49).

La selección de todos los pacientes con medicación antipsicótica es una estrategia importante para establecer una línea de base, así como para identificar cambios tempranos en el peso (3). La aplicación diligente de una guía ayudará a los profesionales de la salud a revertir la prevalencia de la obesidad con el fin de responder a los objetivos de Healthy People 2020
(49). Entre los objetivos, los médicos son llamados a desempeñar un rol activo en la promoción de problemas de control de peso entre sus pacientes.

Las guías basadas en la evidencia para detectar y tratar la obesidad están disponibles para su uso por los profesionales de la salud (49). La guía de la USPSTF proporciona estrategias para identificar, tratar y controlar la obesidad basados en evidencias actuales, incluyendo los programas más efectivos de ejercicio, dieta y terapia conductual. Los profesionales de la salud en todas las especialidades médicas necesitan guías precisas y mejores herramientas de detección para controlar la obesidad (31).

\section{DISCUSIÓN}

Las PVTM presentan un mayor riesgo de desarrollar obesidad, en comparación con la población general. Dentro de los factores identificados se destaca la situación de vulnerabilidad social, la mala alimentación, baja actividad física y el uso de medicamentos antipsicóticos $(28,50)$. Como se ha mostrado, las personas PVTM fallecen antes que la población general, siendo los problemas cardiovasculares los principales responsables. Se han desarrollado intervenciones para mejorar los estilos de vida, pero en su mayoría han sido creadas específicamente para tal fin, con muestras pequeñas y sin tener en cuenta resultados de la población general (51); por tanto, los resultados deben tomarse con precaución, aunque se muestren esperanzadores.

Modificar la dieta combinada con ejercicios estructurados y terapia conductual puede reducir significativamente la hipertensión y disminuir el riesgo de síndrome metabólico (52). Estas intervenciones son particularmente importantes en el contexto de PVTM.

Esta revisión reveló escasa evidencia sobre el nivel de educación de los profesionales de la salud mental con respectoa la identificación, prevención y manejo de la obesidad en personas que viven con trastornos mentales. Los resultados indican que los profesionales que trabajan en salud mental están utilizando enfoques obsoletos para controlar la obesidad en personas que padecen trastornos mentales.

Los profesionales de salud mental están en una buena posición para abordar la obesidad utilizando estrategias para revertir el aumento de peso con cambios en el estilo de vida, así como el manejo de medicamentos psiquiátricos. Estas 
estrategias contribuyen a disminuir la morbilidad y aumentar la calidad de vida (52).

Los profesionales de área deben empezar a evaluar tanto la nutrición como actividad física de las PVTM en sus rutinas asistenciales. Estrategias basadas en la evidencia para el manejo del peso incluyen intervenciones dietéticas y actividad física los que han mostrado resultados prometedores. Fernández-San-Martin et al. (53) indican en su meta-análisis, que este tipo de intervenciones disminuyen los valores bioquímicos y antropométricos a los 3, 6 y 12 meses. Otros autores reportan mejoría pasados hasta 18 meses tras la intervención (37). En definitiva, un manejo adecuado y un compromiso médico-paciente produce resultados positivos para el paciente, comunidad y sociedad.

El ser consciente del problema en conjunto con el acceso a las estrategias basadas en la evidencia para abordar el problema resultan ser importantes para revertir la obesidad en PVTM. Las PVTM reciben atención limitada por parte de los psiquiatras en cuestiones relacionadas con el aumento de peso e intervención (37). Sin embargo, cuando a las PVTM se les brinda la oportunidad de participar en un programa formal de control de peso, en promedio pueden lograr una pérdida significativa del mismo (22).

La educación basada en la evidencia relacionada con el manejo de la obesidad debe convertirse en un aspecto clave del manejo clínico y plan de cuidado.

\section{CONCLUSIONES}

Los índices de obesidad entre las PVTM se encuentran en niveles epidémicos y varían según la gravedad de la enfermedad. El riesgo de obesidad debe ser considerado en el manejo clínico de las PVTM. Los profesionales de la salud mental deben ser conscientes respecto a la asociación entre trastornos mentales y alteraciones por exceso de peso. Esta población necesita ser evaluada, monitoreada y manejada precozmente cuando se encuentran en estado de sobrepeso e identificada para manejo intensivo cuando se encuentre en estado de obesidad.

En general, los profesionales de la salud mental pasan por alto los factores de riesgo de la obesidad entre las PVTM, ya que se enfocan en el tratamiento de los trastornos de la salud mental. La educación basada en la evidencia relacionada con el manejo de la obesidad es un aspecto clave para mejorar el manejo clínico y los planes de cuidado. Además, la literatura no cuenta con un enfoque en dietistas y nutricionistas y esto indica que no están involucrados o que no están invitados a participar en la planificación del cuidado y el proceso de manejo. Un objetivo importante para futuras investigaciones es comprender las percepciones y prácticas específicas para la participación de equipo interdisciplinario en el manejo de las PVTM. Adicionalmente, es necesario que existan programas de desarrollo profesional que incrementen la conciencia de los profesionales de salud mental sobre la oculta epidemia de obesidad en su población de pacientes. Finalmente, las guías de práctica clínica basadas en la evidencia necesitan ser desarrolladas y así incrementar la colaboración a los profesionales de la salud mental con enfermeras, fisioterapeutas, dietistas y nutricionistas, en el manejo integral de sus pacientes.

\section{REFERENCIAS BIBLIOGRÁFICAS}

1. Centers for Disease Control and Prevention. Adult obesity: obesity rises among adults 2010 [updated August 3, 2010]. Available from:

https://www.cdc.gov/vitalsigns/AdultObesit y/index.html.

2. Khalil RB. Metabolic syndrome in patients with schizophrenia independently from atypical antipsychotics intake. Presse Med. 2012;41(5):e238-e43. https://doi.org/10.1016/j.lpm.2011.10.013

3. De Hert M, Cohen D, Bobes J, CetkovichBakmas M, Leucht S, Ndetei DM, et al. Physical illness in patients with severe mental disorders. II. Barriers to care, monitoring and treatment guidelines, plus recommendations at the system and individual level. World Psychiatry. 2011;10(2):138-51. https://doi.org/10.1002/j.20515545.2011.tb00036.x

4. McKibbin CL, Kitchen KA, Wykes TL, Lee AA. Barriers and facilitators of a healthy lifestyle among persons with serious and persistent mental illness: perspectives of community mental health providers. Community Ment Health J. 2014;50(5):56676. https://doi.org/10.1007/s10597-0139650-2

5. Kokkinos P, Panagiotakos DB, Polychronopoulos E. Dietary influences on blood pressure: the effect of the Mediterranean diet on the prevalence of hypertension. J Clin Hypertens. 2005;7(3):165-70. 
https://doi.org/10.1111/j.1524-

6175.2005.04079.x

6. Carra G, Bartoli F, Carretta D, Crocamo C, Bozzetti A, Clerici M, et al. The prevalence of metabolic syndrome in people with severe mental illness: a mediation analysis. Soc Psychiatry Psychiatr Epidemiol. 2014;49(11):1739-46. https://doi.org/10.1007/s00127-014-0835-y

7. Yazici MK, Anil Yagcioglu AE, Ertugrul A, Eni N, Karahan S, Karaagaoglu E, et al. The prevalence and clinical correlates of metabolic syndrome in patients with schizophrenia: findings from a cohort in Turkey. Eur Arch Psychiatry Clin Neurosci. 2011;261(1):69-78. https://doi.org/10.1007/s00406-010-0118-x

8. Sicras-Mainar A, Blanca-Tamayo M, RejasGutiérrez J, Navarro-Artieda R. Metabolic syndrome in outpatients receiving antipsychotic therapy in routine clinical practice: A cross-sectional assessment of a primary health care database. Eur Psychiatry. 2008;23(2):100-8. https://doi.org/10.1016/j.eurpsy.2007.07.00 5

9. Nemiary D, Shim R, Mattox G, Holden K. The relationship between obesity and depression among adolescents. Psychiatr Ann. 2012;42(8):305-8. https://doi.org/10.3928/0048571320120806-09

10. Katekaru M, Minn CE, Pobutsky AM. Weight reduction among people with severe and persistent mental illness after health behavior counseling and monitoring. Hawaii J Med Public Health. 2015;74(4):146-9.

11. Moher D, Stewart L, Shekelle P. All in the family: systematic reviews, rapid reviews, scoping reviews, realist reviews, and more. Syst Rev. 2015;4(183):1-2. https://doi.org/10.1186/s13643-015-0163-7

12. Peters MD, Godfrey CM, Khalil H, McInerney P, Parker D, Soares CB. Guidance for conducting systematic scoping reviews. Int $\mathrm{J}$ Evid Based Healthc. 2015;13(3):141-6. https://doi.org/10.1097/XEB.000000000000 0050

13. Colquhoun HL, Levac D, O'Brien KK, Straus S, Tricco AC, Perrier L, et al. Scoping reviews: time for clarity in definition, methods, and reporting. J Clin Epidemiol. 2014;67(12):1291-4.

https://doi.org/https://doi.org/10.1016/j.jclin epi.2014.03.013
14. Arksey H, O'Malley L. Scoping studies: towards a methodological framework. Int $\mathbf{J}$ Soc Res Methodol. 2005;8(1):19-32. https://doi.org/10.1080/1364557032000119 616

15. Levac D, Colquhoun H, O'Brien KK. Scoping studies: advancing the methodology. Implement Sci. 2010;5(69):19. https://doi.org/10.1186/1748-5908-5-69

16. Newhouse RP, Dearholt SL, Poe SS, Pugh LC, White KM. Johns Hopkins nursing evidence-based practice model and guidelines. Indianapolis, IN: Sigma Theta Tau International; 2007.

17. Tricco A, Straus S, Moher D. Preferred reporting items for systematic reviews and meta-analysis: extension for scoping reviews (PRISMA-ScR): EQUATOR Network; 2015 [Available from: http://www.equatornetwork.org/wpcontent/uploads/2009/02/Executivesummary_ScR_Dec-9.pdf.

18. Galletly CL, Murray LE. Managing weight in persons living with severe mental illness in community settings: a review of strategies used in community interventions. Issues Ment Health Nurs. 2009;30(11):660-8.

19. Piatt EE, Munetz MR, Ritter C. An examination of premature mortality among decedents with serious mental illness and those in the general population. Psychiatr Serv. 2010;61(7):663-8. https://doi.org/10.1176/ps.2010.61.7.663

20. Sartorius N, Holt RIG, Maj M, editors. Comorbidity of mental and physical diseases. Basel, Switzerland: Karger Publishers; 2015.

21. Chacon F, Mora F, Gervas-Rios A, Gilaberte I. Efficacy of lifestyle interventions in physical health management of patients with severe mental illness. Ann Gen Psychiatry. 2011;10:22. https://doi.org/10.1186/1744$859 \mathrm{X}-10-22$

22. Brown C, Stoffel VC, editors. Occupational therapy in mental health: a vision for participation. 1 ed. Philadelphia, PA: F. A. Davis Company; 2011.

23. Capodaglio P, Faintuch J, Liuzzi A, editors. Disabling obesity: from determinants to health care models. 1 ed. Berlin, Germany: Springer Science \& Business Media; 2013.

24. Yatham LN, Malhi GS. Bipolar disorder. New York, NY: Oxford University Press; 2011.

25. Yildiz A, Ruiz P, Nemeroff CB, editors. The bipolar book: history, neurobiology, and 
treatment. New York, NY: Oxford University Press; 2015.

26. Hardy S, White J, Gray RJ, editors. The health improvement profile (HIP): a manual to promote physical well-being in people with severe mental illness. Cumbria, CA: M\&K Publishing; 2015.

27. de Wit L, Luppino F, van Straten A, Penninx B, Zitman F, Cuijpers P. Depression and obesity: a meta-analysis of community-based studies. Psychiatry Res. 2010;178(2):230-5. https://doi.org/10.1016/j.psychres.2009.04.0 15

28. Bradshaw T, Mairs H. Obesity and serious mental ill health: a critical review of the literature. Healthcare. 2014;2(2):166-82. https://doi.org/10.3390/healthcare2020166

29. Udo I, Mooney M, Newman A. Prevalence of obesity and metabolic syndrome in a longstay psychiatric unit. Irish Journal of Psychological Medicine. 2011;28(4):205-8. https://doi.org/10.1017/S079096670001166 6

30. Green CA, Janoff SL, Yarborough BJ, Yarborough MT. A 12-week weight reduction intervention for overweight individuals taking antipsychotic medications. Community Ment Health J. 2014;50(8):974-80.

https://doi.org/10.1007/s10597-014-9716-9

31. Epling JW, Morley CP, Ploutz-Snyder R. Family physician attitudes in managing obesity: a cross-sectional survey study. BMC Res Notes. 2011;4(473):1-8. https://doi.org/10.1186/1756-0500-4-473

32. Lichwala-Zyla C, Price JH, Dake JA, Jordan T, Price JA. Psychiatrists' perceptions and practices in treating patients' obesity. Acad Psychiatry. 2009;33(5):370-6. https://doi.org/10.1176/appi.ap.33.5.370

33. Sugawara N, Yasui-Furukori N, Yamazaki M, Shimoda K, Mori T, Sugai T, et al. Psychiatrists' attitudes toward metabolic adverse events in patients with schizophrenia. PLoS One. 2014;9(1):e86826. https://doi.org/10.1371/journal.pone.008682 6

34. Stanford FC, Johnson ED, Claridy MD, Earle RL, Kaplan LM. The role of obesity training in medical school and residency on bariatric surgery knowledge in primary care physicians. Int J Family Med. 2015;2015(841249):1-8. https://doi.org/10.1155/2015/841249

35. Sebiany AM. Primary care physicians' knowledge and perceived barriers in the management of overweight and obesity. J Family Community Med. 2013;20(3):14752. 8229.121972

36. Bleich SN, Bandara S, Bennett WL, Cooper LA, Gudzune KA. U.S. health professionals' views on obesity care, training, and selfefficacy. Am J Prev Med. 2015;48(4):411-8. https://doi.org/10.1016/j.amepre.2014.11.00 2

37. Daumit GL, Dickerson FB, Wang NY, Dalcin A, Jerome GJ, Anderson CA, et al. A behavioral weight-loss intervention in persons with serious mental illness. N Engl J Med. 2013;368(17):1594-602. https://doi.org/10.1056/NEJMoa1214530

38. Hardy S, White J, Deane K, Gray R. Educating healthcare professionals to act on the physical health needs of people with serious mental illness: a systematic search for evidence. J Psychiatr Ment Health Nurs. 2011;18(8):721-7. https://doi.org/10.1111/j.13652850.2011.01722.x

39. Smith S, Seeholzer EL, Gullett H, Jackson B, Antognoli E, Krejci SA, et al. Primary care residents' knowledge, attitudes, self-efficacy, and perceived professional norms regarding obesity, nutrition, and physical activity counseling. J Grad Med Educ. 2015;7(3):388-94. https://doi.org/10.4300/JGME-D-1400710.1

40. Mazur A, Matusik P, Revert K, Nyankovskyy S, Socha P, Binkowska-Bury $\mathrm{M}$, et al. Childhood obesity: knowledge, attitudes, and practices of European pediatric care providers. Pediatrics. 2013;132(1):e100-e8. https://doi.org/10.1542/peds.2012-3239

41. Brown C, Goetz J, Hamera E, Gajewski B. Treatment response to the RENEW weight loss intervention in schizophrenia: impact of intervention setting. Schizophr Res. 2014;159(2-3):421-5. https://doi.org/10.1016/j.schres.2014.09.018

42. Bonfioli E, Berti L, Goss C, Muraro F, Burti L. Health promotion lifestyle interventions for weight management in psychosis: a systematic review and meta-analysis of randomised controlled trials. BMC Psychiatry. 2012;12:78. https://doi.org/10.1186/1471-244X-12-78

43. Naslund JA, Aschbrenner KA, Scherer EA, Pratt SI, Wolfe RS, Bartels SJ. Lifestyle intervention for people with severe obesity and serious mental illness. Am J Prev Med. 
2016;50(2):145-53. https://doi.org/10.1016/j.amepre.2015.07.01 2

44. Brown S, Kim M, Mitchell C, Inskip H. Twenty-five year mortality of a community cohort with schizophrenia. Br J Psychiatry. 2010;196(2):116-21. https://doi.org/10.1192/bjp.bp.109.067512

45. Megna JL, Schwartz TL, Siddiqui UA, Herrera Rojas M. Obesity in adults with serious and persistent mental illness: a review of postulated mechanisms and current interventions. Ann Clin Psychiatry. 2011;23(2):131-40.

46. Welsh JA, Nelson JM, Walsh S, Sealer H, Palmer W, Vos MB. Brief training in patientcentered counseling for healthy weight management increases counseling selfefficacy and goal setting among pediatric primary care providers: results of a pilot program. Clin Pediatr (Phila). 2015;54(5):425-9. https://doi.org/10.1177/0009922814553432

47. Zhu DQ, Norman IJ, While AE. Nurses' selfefficacy and practices relating to weight management of adult patients: a path analysis. Int $\mathrm{J}$ Behav Nutr Phys Act. 2013;10(131):1-11. https://doi.org/10.1186/1479-5868-10-131

48. Sherson EA, Yakes Jimenez E, Katalanos N. A review of the use of the 5 A's model for weight loss counselling: differences between physician practice and patient demand. Fam Pract. 2014;31(4):389-98. https://doi.org/10.1093/fampra/cmu020

49. United States Preventive Services Task Force. Screening for obesity in adults: recommendations and rationale. Ann Intern Med. 2003;139(11):930-2. https://doi.org/10.7326/0003-4819-139-11200312020-00012

50. Shrivastava A, Johnston ME. Weight-gain in psychiatric treatment: risks, implications, and strategies for prevention and management. Mens Sana Monogr. 2010;8(1):53-68. https://doi.org/10.4103/0973-1229.58819

51. Ward MC, White DT, Druss BG. A metareview of lifestyle interventions for cardiovascular risk factors in the general medical population: lessons for individuals with serious mental illness. J Clin Psychiatry. 2015;76(4):e477-e86. https://doi.org/10.4088/JCP.13r08657

52. Radke AQ, Parks J, Ruter TJ. A call for improved prevention and reduction of obesity among persons with serious mental illness. Psychiatr Serv. 2010;61(6):617-9. https://doi.org/10.1176/ps.2010.61.6.617

53. Fernandez-San-Martin MI, Martin-Lopez LM, Masa-Font R, Olona-Tabuena N, Roman Y, Martin-Royo J, et al. The effectiveness of lifestyle interventions to reduce cardiovascular risk in patients with severe mental disorders: meta-analysis of intervention studies. Community Ment Health J. 2014;50(1):81-95. https://doi.org/10.1007/s10597-013-9614-6 